\title{
ANCHORING THE DISTANCE SCALE VIA X-RAY/INFRARED DATA FOR CEPHEID CLUSTERS: SU Cas
}

\author{
D. Majaess ${ }^{1}$, D. G. Turner ${ }^{1}$, L. Gallo ${ }^{1}$, W. Gieren ${ }^{2}$, C. Bonatto ${ }^{3}$, D. J. Lane ${ }^{1}$, D. Balam ${ }^{4}$, And L. Berdnikov ${ }^{5,6}$ \\ ${ }^{1}$ Department of Astronomy and Physics, Saint Mary's University, Halifax, NS B3H 3C3, Canada; dmajaess@ap.smu.ca \\ ${ }^{2}$ Departamento de Astronomía, Universidad de Concepción, Casilla 160-C, CL Concepción, Chile \\ ${ }^{3}$ Departamento de Astronomía, Universidade Federal do Rio Grande do Sul, Av. Bento Gonçalves, 9500 Porto Alegre, RS, Brazil \\ ${ }^{4}$ Dominion Astrophysical Observatory, Victoria, BC, Canada \\ ${ }^{5}$ Moscow M V Lomonosov State University, Sternberg Astronomical Institute, Moscow 119992, Russia \\ ${ }^{6}$ Isaac Newton Institute of Chile, Moscow Branch, Universitetskij Pr. 13, Moscow 119992, Russia \\ Received 2012 March 15; accepted 2012 May 8; published 2012 June 22
}

\begin{abstract}
New X-ray (XMM-Newton) and $J H K_{s}$ (Observatoire du Mont-Mégantic) observations for members of the star cluster Alessi 95, which Turner et al. discovered hosts the classical Cepheid SU Cas, were used in tandem with UCAC3 (proper motion) and Two Micron All Sky Survey observations to determine precise cluster parameters: $E(J-H)=0.08 \pm 0.02$ and $d=405 \pm 15 \mathrm{pc}$. The ensuing consensus among cluster, pulsation, and trigonometric distances $\left(d=414 \pm 5\left(\sigma_{\bar{x}}\right) \pm 10(\sigma)\right.$ pc) places SU Cas in a select group of nearby fundamental Cepheid calibrators ( $\delta \mathrm{Cep}, \zeta \mathrm{Gem}$ ). High-resolution X-ray observations may be employed to expand that sample as the data proved pertinent for identifying numerous stars associated with SU Cas. Acquiring X-ray observations of additional fields may foster efforts to refine Cepheid calibrations used to constrain $H_{0}$.
\end{abstract}

Key words: Hertzsprung-Russell and C-M diagrams - infrared: stars - open clusters and associations: general stars: variables: Cepheids - X-rays: stars

Online-only material: color figures

\section{INTRODUCTION}

Turner et al. (2012) discovered that the $1.95^{\mathrm{d}}$ classical Cepheid SU Cas is a member of the star cluster Alessi 95. The discovery permitted the fundamental parameters $\left(\log \tau, \mathcal{M}_{*} / M_{\odot}, E_{B-V}, M_{V}, R_{*} / R_{\odot}\right)$ for SU Cas to be inferred from cluster membership, thereby enabling a long-standing debate concerning the Cepheid's distance and pulsation mode to be resolved (Gieren 1976, 1982; Evans 1991; Usenko et al. 2001; Turner et al. 2012 and references therein). Previous uncertainties associated with SU Cas permeated into efforts to anchor the short-period regime of the Cepheid period-luminosity relation. SU Cas is the vital link since the variable is among the shortest-period Galactic Cepheids known (Berdnikov 2008), and the nearest such star (Berdnikov et al. 2000). ${ }^{7}$ The Cepheid's proximity allows for independent confirmation of its distance via trigonometric parallax (Hipparcos; Perryman \& ESA 1997; van Leeuwen 2007; Turner et al. 2012). A principal research objective is to establish Cepheid calibrators with distances secured by independent means: e.g., infrared surface brightness technique (ISB; Fouque \& Gieren 1997; Gieren et al. 2005; Barnes 2009; Storm et al. 2011), cluster membership (Turner 2010; Majaess et al. 2011c), IUE-binary (Evans 1992, 1995), and trigonometric parallaxes (van Leeuwen 2007; Benedict et al. 2007). Achievement of that goal will facilitate efforts by the Carnegie Hubble (Freedman \& Madore 2010) and $\mathrm{SH}_{0} \mathrm{ES}$ (Macri \& Riess 2009) projects to determine $H_{0}$ reliably, and break degeneracies complicating the selection of a cosmological model (Riess et al. 2011). Incidentally, the prototype of the class $(\delta \mathrm{Cep})$ has the most precise Cepheid distance established $\left(d=272 \pm 3\left(\sigma_{\bar{x}}\right) \pm 5(\sigma) \mathrm{pc}\right)$, which is tied to a cluster distance from $U B V J H K_{s}$ photometry (Majaess et al. 2012a), Hipparcos trigonometric parallaxes for cluster stars

\footnotetext{
7 Polaris is nearer (Turner 2009; Turner et al. 2012), yet exhibits a pulsation period $\sim 2 \times$ larger than SU Cas (Berdnikov 2008). However, that does not mitigate Polaris' importance for the distance scale.
}

(de Zeeuw et al. 1999; van Leeuwen 2007; Majaess et al. 2012a), and Hipparcos /Hubble Space Telescope (HST) trigonometric parallaxes for the Cepheid and its companion HD213307 ${ }^{8}$ (Benedict et al. 2002; van Leeuwen 2007). $\zeta$ Gem is another nearby Cepheid which exhibits a precise mean distance $\left(d=363 \pm 9\left(\sigma_{\bar{x}}\right) \pm 26(\sigma)\right.$ pc; Majaess et al. 2012b) tabulated from cluster membership, HST/Hipparcos parallaxes for the Cepheid and cluster stars, and an ISB distance (Gieren et al. 2005; Storm et al. 2011). The desire to incorporate SU Cas into that select sample of fundamental Cepheid calibrators is the impetus for this research (see also Turner et al. 2012).

In this study, new X-ray and $J H K_{s}$ data from XMM-Newton (Jansen et al. 2001) and the Observatoire du Mont-Mégantic (OMM; Artigau et al. 2010) are presented for Alessi 95. X-ray observations provide a means of segregating cluster members from field stars (Randich et al. 1996; Evans 2011), while OMM data supply more reliable photometry for fainter stars than Two Micron All Sky Survey (2MASS; Cutri et al. 2003).

\section{ANALYSIS}

\subsection{Brighter Cluster Members}

The positions for stars $r<35^{\prime}$ from SU Cas that feature UCAC3 (Third U.S. Naval Observatory CCD Astrograph Catalog; Zacharias et al. 2010) proper motions and 2MASS photometry are shown in Figure 1 (top panel). The stellar overdensity near the proper motion for SU Cas $\left(\mu_{\alpha}=1.0 \pm 0.7\right.$ and $\mu_{\delta}=-8.6 \pm 0.8 \mathrm{mas} \mathrm{yr}^{-1}$ ) represents the cluster Alessi 95. The analysis was subsequently expanded and 45 stars with similar proper motions to SU Cas $\left(\mu_{\alpha}=-0.5 \rightarrow 5.0\right.$ and


tected within $\sim 1^{\circ}$ of the Cepheid. The positions and proper motions of those objects are displayed in Figure 1 (bottom

\footnotetext{
8 XMM-Newton data confirm that HD213307 features a companion (Benedict et al. 2002). $\delta$ Cep is thus a multiple system, somewhat analogous to Polaris (Evans et al. 2010).
} 

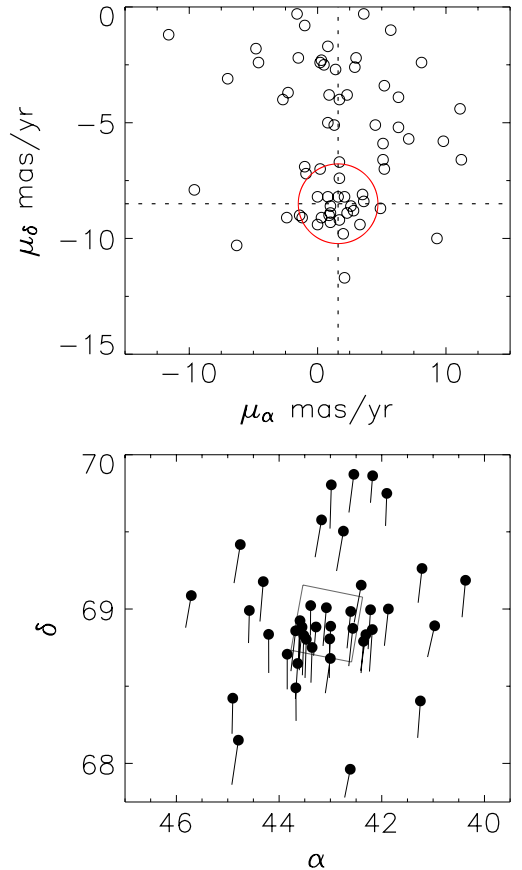

Figure 1. Top panel: UCAC3 proper motions for brighter stars within $r \sim 35^{\prime}$ of SU Cas. Cluster members aggregate near the proper motion estimate for SU Cas $\left(\mu_{\alpha}: \mu_{\delta}=1.0 \pm 0.7:-8.6 \pm 0.8 \mathrm{mas}^{-1}\right)$. Field contamination becomes more acute with increasing distance from the cluster center, thus the sample was restricted to $r \leqslant 35^{\prime}$ to highlight the cluster's significance. Bottom panel: the cluster core is discernible in the spatial distribution of stars $r \leqslant 60^{\prime}$ from SU Cas which exhibit $\mu_{\alpha}=-0.5 \rightarrow 5.0$ and $\mu_{\delta}=-6.0 \rightarrow-10.5 \mathrm{mas} \mathrm{yr}^{-1}$ $\left(\sigma<5 \mathrm{mas} \mathrm{yr}^{-1}\right)$. Proper motion trajectories $\left(10^{5} \mathrm{yr}\right)$ are overplotted merely for illustrative purposes. The area bounded by the solid lines represents the approximate region sampled by XMM-Newton.

(A color version of this figure is available in the online journal.)

panel), and the cluster core is discernible in that diagram. Of the proper motion selected stars within $r \lesssim 40^{\prime}$ of SU Cas (Figure 1), 27 form a distinct main sequence in the $J H$ color-magnitude diagram (Figure 3). Only one star from the proper motion selected sample (Figure 1 ) exhibiting $r \lesssim 40^{\prime}$ appears to be a non-member, reaffirming that the proper motion data efficiently separate cluster members from field stars (Figures 1 and 3). An inability to distinguish cluster members

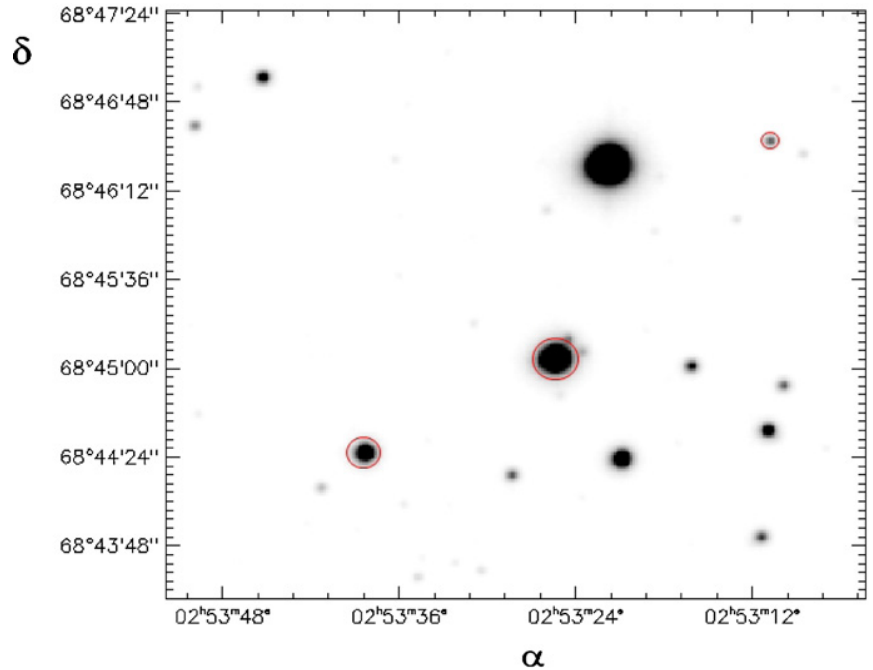

Figure 2. Finder chart (OMM $J$ ) for three probable cluster members which are $\mathrm{X}$-ray sources. The two brighter objects exhibit UCAC3 proper motions (e.g.,


Cas $\left(\mu_{\alpha}: \mu_{\delta}=1.0 \pm 0.7:-8.6 \pm 0.8 \mathrm{mas}^{-1}\right)$. The stars are probable cluster members based on X-ray, proper motion, and color-magnitude data (Figure 3). The finder chart represents a small portion of the observed field.

(A color version of this figure is available in the online journal.)

from field stars hinders isochrone fitting and exacerbates uncertainties tied to the derived distance. For example, the host cluster for $\zeta$ Gem proved more difficult to assess owing to the small offset of its proper motion relative to the field, particularly given the uncertainties (Majaess et al. 2012b). That shortcoming may be addressed by acquiring XMM-Newton/Chandra observations to identify cluster members, as later-type stars associated with $\zeta$ Gem $(\log \tau=7.85 \pm 0.15$; Majaess et al. 2012a) may emit X-rays owing to their comparative youth (Randich et al. 1996; Evans et al. 2010; Evans 2011). Independent confirmation of the $\zeta$ Gem cluster (Majaess et al. 2012a) is desirable.

$\mathrm{X}$-ray data for the SU Cas field (PI: Guinan) were obtained from the XMM-Newton public data archive (XSA). Point sources identified via the XMM-Newton Processing Pipeline Subsystem (PPS; Figure 2) were correlated with 2MASS photometry (closest source within 0.1). The majority of the X-ray sources

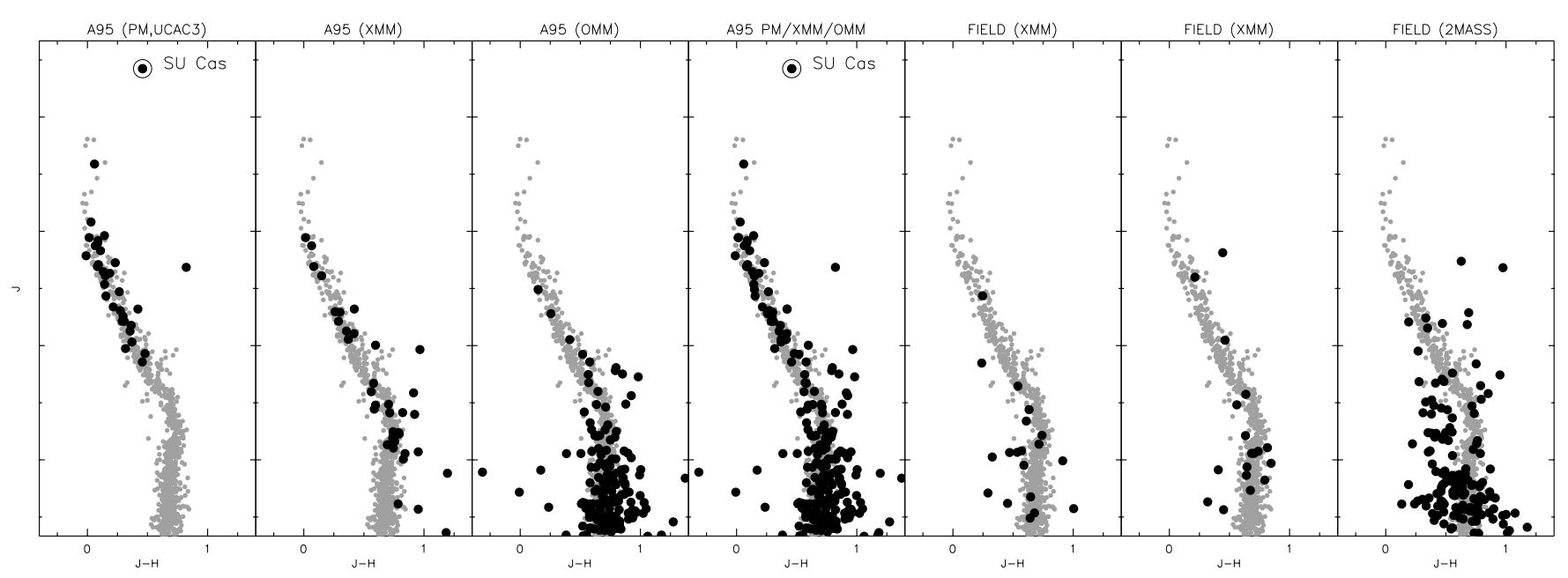

Figure 3. $J H$ color-magnitude diagrams for stars exhibiting similar proper motions to SU Cas (PM, UCAC3), stars emitting X-rays (XMM), and OMM stars sampled $r<4^{\prime}$ from the center of Alessi 95 (A95). Gray dots are calibration stars from Majaess et al. (2011b). Comparison fields sampled by XMM-Newton and 2MASS imply that the cluster (A95) distribution is not fortuitous. 
lie directly on the cluster main sequence (Figure 3), and the field stars are readily discernible in the diagram (e.g., objects redder than M-type cluster dwarfs). X-ray observations are a pertinent means for identifying cluster members in harmony with other methods. The brightest star in Figure 2 (BD+68 201, B9III-IV; Turner et al. 2012) may exhibit a later-type companion which is the source of the X-ray emission, since later-type B stars are typically X-ray quiet (Evans 2011 and references therein). $\mathrm{BD}+68^{\circ} 201$ and SU Cas exhibit analogous UCAC3 proper motions, to within the uncertainties. The faintest star in Figure 2 is likely an M-type cluster dwarf, as implied by its $J H K_{S}$ photometry. A list of the X-ray sources and their corresponding 2MASS data are available via the XMM-Newton public data archive (PPS products).

\subsubsection{Mean Reddening for Alessi 95}

A $J H K_{s}$ color-color analysis of the UCAC3/XMM sample was performed to determine the mean reddening. The $J H K_{s}$ intrinsic relations of Straižys \& Lazauskaite (2009) and Turner (2011) were employed to infer a mean color excess of $E(J-H)=0.08 \pm 0.02$. That result is tied to a reddening law of $E(J-H) / E\left(H-K_{s}\right)=2.0$ (Straižys \& Lazauskaite 2009; Majaess et al. 2011c). Stars that lie above the main sequence (potential binary systems) in the color-magnitude diagram were excluded from the determination, in tandem with other stars exhibiting anomalous $J H K_{s}$ colors. The mean color excess determined agrees with the optical excess established by Laney \& Caldwell (2007), Kovtyukh et al. (2008), and Turner et al. (2012; see also Table 5 in Evans 1991 and references therein). The conversion from the infrared to optical reddening is somewhat uncertain (Bonatto et al. 2004; Majaess et al. 2008, and references therein). Therefore, the optical reddening $\left(E_{B-V} \sim 0.30 \pm 0.05\right)$ derived from $E(J-H)$ exhibits larger uncertainty to reflect that ambiguity.

\subsection{Fainter Cluster Members}

Deeper $J H K_{S}$ images were obtained for the SU Cas field from the OMM. The OMM houses a $1.6 \mathrm{~m}$ telescope equipped with a near-infrared wide-field imager $\left(\mathrm{CPAPIR}^{9}\right)$. The images were acquired on 2012 January 11. Point spread function (PSF) photometry was performed using DAOPHOT (Stetson 1987). The instrumental photometry was subsequently tied to 2MASS secondary standards in the field. A color-magnitude diagram was tabulated for stars within $r<4^{\prime}$ of the cluster center to mitigate field contamination. OMM $J H K_{s}$ photometry for stars near the core of Alessi 95 shall be tabulated online at CDS or WEBDA (Paunzen 2008).

Comparison fields displayed in Figure 3 reaffirm the existence of Alessi 95. However, the analysis is complicated by the cluster's extent, high latitude position $\left(\ell, b \sim 133^{\circ}, 9^{\circ}\right)$ and inhomogeneous reddening. The first XMM-Newton comparison field lies beyond the coronal radius $(02: 05: 55+64: 56: 33, r \sim$ $6^{\circ}$ ); however, hierarchical clustering observed in giant molecular clouds implies that members may still be sampled. The second $X M M-N e w t o n$ comparison field encompasses another classical Cepheid ( $\beta$ Dor). The 2MASS comparison field (Figure 3, far right panel) is equal in area to the $r \sim 4^{\prime}$ OMM sampling, but traces a symmetric annulus $r \sim 5^{\circ}$ away. Field contamination will be most acute for the fainter OMM data.

No low-mass stars are visible in HST WFC3 images for SU Cas (HST Proposal 12215; Evans 2009). The saturation

\footnotetext{
9 http://www.astro.umontreal.ca/cpapir
}

from the Cepheid was treated by subtracting (normalized) the image from a master, which was constructed (median combine) using numerous Cepheids observed for proposal 12215 (see also Evans 2011). The late B-type (B9.5V) companion discovered by Evans (1991, their Figure 4) via IUE observations was not detected, indicating that the star is in rather close proximity to the Cepheid. A comprehensive analysis is forthcoming (N. R. Evans et al., in preparation).

\subsection{Distance to Alessi 95}

A precise distance may be established for the cluster via mainsequence fitting. The infrared (2MASS/NOMAD) calibration presented by Majaess et al. (2011b) was employed since it offers numerous advantages. First, the $J H K_{s}$ calibration is comparatively insensitive to variations in $[\mathrm{Fe} / \mathrm{H}]$ and age (Majaess et al. 2011b; see also Straižys \& Lazauskaitė 2009). Second, the deep $J H K_{s}$ photometry extends into the low-mass regime $\left(\simeq 0.4 M_{\odot}\right)$ where $J-K_{s}$ remains constant with increasing magnitude $\left(M_{J} \gtrsim 6\right)$ for low-mass M-type dwarfs, and $J-H$ exhibits an inversion (see Figure 3; Majaess et al. 2011b and references therein). The trends ensure precise $J H K_{s}$ main-sequence fits by providing distinct anchor points in color-magnitude and color-color diagrams (Figure 3). Third, the calibration's zero point is tied to seven benchmark open clusters $(d<250 \mathrm{pc})$ which exhibit matching $J H K_{s}$ and revised Hipparcos distances (the Hyades, $\alpha$ Per, Praesepe, Coma Ber, IC 2391, IC 2609, and NGC 2451; van Leeuwen 2009; Majaess et al. 2011b; see also McArthur et al. 2011). The scale is anchored using clusters where consensus exists, rather than the discrepant case (i.e., the Pleiades). The objective is to avoid deriving distances to Cepheid clusters using a single benchmark cluster, and prevent the propagation of ambiguity into the Cepheid calibration. The revised Hipparcos distance for the Pleiades is $d=120.2 \pm 1.9 \mathrm{pc}$ (van Leeuwen 2009), whereas $J H K_{s}$ data implied $d=138 \pm 6 \mathrm{pc}$ (Majaess et al. 2011b), and Soderblom et al. (2005) employed $H S T$ to deduce $d=134.6 \pm 3.1 \mathrm{pc}$. By contrast, van Leeuwen (2009) obtained $d=172.4 \pm 2.7$ pc for $\alpha$ Per, which matches that established via the $J H K_{s}$ analysis (Majaess et al. 2011b, their Table 1). Fourth, potential variations in the $J H K_{s}$ reddening and extinction laws are predicted to be comparatively smaller than in the optical. Obscuration by dust is less significant in the infrared $\left(E_{J-H} \sim 0.3 \times E_{B-V}\right.$; Majaess et al. 2008; Bonatto et al. 2004 and references therein), which consequently mitigates the impact of variations in $R_{\lambda}\left(J_{0}=J-E_{J-H} \times R_{J}\right)$. The ratio of total to selective extinction $R_{J}$ was adopted from Majaess et al. (2011c; see also Bonatto et al. 2004). The offset from the calibration yields a distance to Alessi 95 of $d=405 \pm 15 \mathrm{pc}$.

\subsection{Distance to $S U$ Cas}

A mean distance to SU Cas may be derived from the new $J H K_{s}$ cluster parameters established here (the Cepheid lies near cluster center), and the following estimates: the distance inferred from $U B V$ photometry and optical spectra for cluster members $(d=429 \pm 8$ pc; Turner et al. 2012), the mean Hipparcos parallax for cluster stars ( $420 \pm 33$ pc; van Leeuwen 2007; Turner et al. 2012), the ISB distance for SU Cas ( $d=414 \pm 12$ pc; Storm et al. 2011; Turner et al. 2012), the original Hipparcos Cepheid parallax $(d=433 \pm 116$ pc; Perryman \& ESA 1997), and the revised Hipparcos Cepheid parallax $(d=395 \pm 50$ pc; van Leeuwen 2007). A weighted mean of $d=414 \pm 5\left(\sigma_{\bar{x}}\right) \pm 10(\sigma)$ pc was established after assigning $w=3$ to the ISB, $J H K_{s}$, and $U B V$ distances, $w=2$ for the revised Hipparcos distances, and 
$w=1$ for the original Hipparcos distance. $\sigma_{\bar{x}}$ is the standard error, $\sigma$ is the standard deviation, and $w$ is the weight. Lastly, the absolute Wesenheit magnitude $\left(W_{V I_{c}}\right)$ for SU Cas implied by that distance should be corrected for contamination from the companion (Evans 1991, 1992; see also Turner et al. 2012).

\section{CONCLUSION AND FUTURE RESEARCH}

New XMM-Newton and OMM $J H K_{s}$ photometry for Alessi 95, in conjunction with existing UCAC3 and 2MASS observations, imply cluster parameters of $E(J-H)=0.08 \pm 0.02$ and $d=405 \pm 15 \mathrm{pc}$ (Figures 1 and 3$)$. The determination confirms the findings by Turner et al. (2012), and in particular that SU Cas is an overtone pulsator (Gieren 1982; Evans 1991). Distance estimates for SU Cas (i.e., ISB, Hipparcos, cluster membership) converge upon $d=414 \pm 5\left(\sigma_{\bar{x}}\right) \pm 10(\sigma) \mathrm{pc}$ (Section 2.4). The small uncertainty places SU Cas in a select group of classical Cepheid calibrators ( $\delta$ Cep and $\zeta$ Gem) that exhibit precise distances owing to cluster membership and the availability of trigonometric parallaxes. Yet the establishment of an HST parallax for SU Cas remains desirable in order to corroborate (or refute) the results. HST fine guidance sensor images are available for SU Cas and were obtained as part of proposal ID 10113 (Bond 2004). Moreover, reducing the UCAC3 proper motion uncertainties and potentially extending the analysis to fainter stars would strengthen the distance derived. Precise proper motions for fainter stars near SU Cas may be obtained from photographic plates stored at the CfA (Grindlay 2007; DASCH). ${ }^{10}$ The plates offer unprecedented multi-epoch coverage spanning a $\sim 100$ year baseline, and uncertainties are further mitigated owing to sizable statistics $\left(\sim(5-10) \times 10^{2}\right.$ plates per object $)$.

Lastly, XMM-Newton X-ray data proved pertinent for segregating cluster members from field stars, thereby highlighting a novel approach for establishing improved distances for cluster Cepheids (see also Evans 2011). Identifying members of Cepheid clusters using X-ray and proper motion data, as demonstrated here for SU Cas, may invariably bolster the Cepheid calibration (e.g., Ngeow 2012) and efforts to establish extragalactic distances (e.g., Shappee \& Stanek 2011; Gerke et al. 2011; Freedman \& Madore 2010). Admittedly, considerable work remains to fine tune the former approach and maximize full use of the available X-ray data. Furthermore, additional work is needed to anchor the long-period end of the Galactic Cepheid calibration, despite continued advancements to secure the short- and intermediate-period regimes. Future research shall aim to assess the viability of establishing long-period Cepheid calibrators from their membership in spiral arms, which are likewise delineated by young open clusters (see Majaess et al. 2011a).

D.M. is grateful to the following individuals and consortia whose efforts lie at the foundation of the research: OMM (E. Artigau, R. Lamontagne, R. Doyon), UCAC3 (N. Zacharias), 2MASS, N. Evans, XMM-Newton (N. Loiseau, R. G. Riestra), E. Guinan, S. Engle, F. van Leeuwen/M. Perryman (Hipparcos), P. Stetson (DAOPHOT), WEBDA (E. Paunzen), DAML (W. Dias), CDS, arXiv, and NASA ADS. W.G. is grateful for support from the Chilean Center for Astrophysics FONDAP 15010003 and the BASAL Centro de Astrofisica y Tecnologias Afines (CATA) PFB-06/2007.

\section{REFERENCES}

Artigau, É., Lamontagne, R., Doyon, R., \& Malo, L. 2010, Proc. SPIE, 7737 Barnes, T. G. 2009, in AIP Conf. Proc. 1170, Stellar Pulsation: Challenges for Theory and Observation, ed. J. A. Guzik \& P. A. Bradley (Melville, NY: AIP), 3

Benedict, G. F., McArthur, B. E., Feast, M. W., et al. 2007, AJ, 133, 1810

Benedict, G. F., McArthur, B. E., Fredrick, L. W., et al. 2002, AJ, 124, 1695

Berdnikov, L. N. 2008, VizieR Online Data Catalog, 2285, 0

Berdnikov, L. N., Dambis, A. K., \& Vozyakova, O. V. 2000, A\&AS, 143, 211

Bonatto, C., Bica, E., \& Girardi, L. 2004, A\&A, 415, 571

Bond, H. 2004, HST Proposal, 10113

Cutri, R. M., Skrutskie, M. F., van Dyk, S., et al. 2003, The IRSA 2MASS All-Sky Point Source Catalog, NASA/IPAC Infrared Science Archive

de Zeeuw, P. T., Hoogerwerf, R., de Bruijne, J. H. J., Brown, A. G. A., \& Blaauw, A. $1999, \mathrm{AJ}, 117,354$

Evans, N. R. 1991, ApJ, 372, 597

Evans, N. R. 1992, ApJ, 389, 657

Evans, N. R. 1995, ApJ, 445, 393

Evans, N. 2009, HST Proposal, 12215

Evans, N. R. 2011, in IAU Symp. 272, Active OB Stars: Structure, Evolution, Mass Loss, and Critical Limits, ed. C. Neiner, G. Wade, G. Meynet, \& G. Peters (Cambridge: Cambridge Univ. Press), 537

Evans, N. R., Guinan, E., Engle, S., et al. 2010, AJ, 139, 1968

Fouque, P., \& Gieren, W. P. 1997, A\&A, 320, 799

Freedman, W. L., \& Madore, B. F. 2010, ARA\&A, 48, 673

Gerke, J. R., Kochanek, C. S., Prieto, J. L., Stanek, K. Z., \& Macri, L. M. 2011, ApJ, 743, 176

Gieren, W. 1976, A\&A, 47, 211

Gieren, W. 1982, PASP, 94, 960

Gieren, W., Storm, J., Barnes, T. G., III, et al. 2005, ApJ, 627, 224

Grindlay, J. E. 2007, in ASP Conf. Ser. 373, The Central Engine of Active Galactic Nuclei, ed. L. C. Ho \& J.-M. Wang (San Francisco, CA: ASP), 711

Jansen, F., Lumb, D., Altieri, B., et al. 2001, A\&A, 365, L1

Kovtyukh, V. V., Soubiran, C., Luck, R. E., et al. 2008, MNRAS, 389, 1336

Laney, C. D., \& Caldwell, J. A. R. 2007, MNRAS, 377, 147

Macri, L. M., \& Riess, A. G. 2009, in AIP Conf. Proc. 1170, Stellar Pulsation: Challenges for Theory and Observation, ed. J. A. Guzik \& P. A. Bradley (Melville, NY: AIP), 23

Majaess, D., Turner, D., \& Gieren, W. 2011a, ApJ, 741, L36

Majaess, D., Turner, D., \& Gieren, W. 2012a, ApJ, 747, 145

Majaess, D., Turner, D., Gieren, W., Balam, D., \& Lane, D. 2012b, ApJ, 748, L9

Majaess, D. J., Turner, D. G., \& Lane, D. J. 2008, MNRAS, 390, 1539

Majaess, D. J., Turner, D. G., Lane, D. J., \& Krajci, T. 2011b, J. Am. Assoc. Var. Star Obs., 39, 219

Majaess, D., Turner, D., Moni Bidin, C., et al. 2011c, ApJ, 741, L27

McArthur, B. E., Benedict, G. F., Harrison, T. E., \& van Altena, W. 2011, AJ, 141,172

Ngeow, C.-C. 2012, ApJ, 747, 50

Paunzen, E. 2008, Contributions of the Astronomical Observatory Skalnate Pleso, 38, 435

Perryman, M. A. C., \& ESA, 1997, The Hipparcos and Tycho Catalogues (ESA SP-1200; Noordwijk: ESA), 1200

Randich, S., Schmitt, J. H. M. M., Prosser, C. F., \& Stauffer, J. R. 1996, A\&A, 305,785

Riess, A. G., Macri, L., Casertano, S., et al. 2011, ApJ, 730, 119

Shappee, B. J., \& Stanek, K. Z. 2011, ApJ, 733, 124

Soderblom, D. R., Nelan, E., Benedict, G. F., et al. 2005, AJ, 129, 1616

Stetson, P. B. 1987, PASP, 99, 191

Storm, J., Gieren, W., Fouqué, P., et al. 2011, A\&A, 534, A94

Straižys, V., \& Lazauskaite, R. 2009, Balt. Astron., 18, 19

Turner, D. G. 2009, in AIP Conf. Proc. 1170, Stellar Pulsation: Challenges for Theory and Observation, ed. J. A. Guzik \& P. A. Bradley (Melville, NY: AIP), 59

Turner, D. G. 2010, Ap\&SS, 326, 219

Turner, D. G. 2011, RevMexAA, 47, 127

Turner, D. G., Majaess, D. J., Lane, D. J., et al. 2012, MNRAS, 422, 2501

Usenko, I. A., Kovtyukh, V. V., Klochkova, V. G., Panchuk, V. E., \& Yermakov, S. V. 2001, A\&A, 367, 831

van Leeuwen, F. 2007, A\&A, 474, 653

van Leeuwen, F. 2009, A\&A, 497, 209

Zacharias, N., Finch, C., Girard, T., et al. 2010, AJ, 139, 2184

10 Digital Access to a Sky Century @ Harvard (DASCH), http://hea-www. harvard.edu/DASCH/. 